\title{
Isolation of multidrug-resistant Salmonella in Singapore
}

\author{
Yee Wei Phoon ${ }^{1}$, MBBS, MRCP, Yuen Yue Candice $\underline{C h a n}^{2}$, MBChB, MRCP, Tze Hsien Koh${ }^{3,4}$, MBChB, D(ABMM)
}

\begin{abstract}
Multidrug-resistant Salmonella is a well-recognised problem worldwide, especially in developing countries such as India, where non-typhoidal Salmonella infections and enteric fever are endemic. Antimicrobial resistance, particularly to fluoroquinolones, is common and leads to the frequent use of alternative agents, such as azithromycin. We herein describe the first reported case of azithromycin-resistant Salmonella gastroenteritis in a Singaporean patient.
\end{abstract}

Keywords: azithromycin, enteric fever, resistance, salmonella

\section{INTRODUCTION}

Salmonella species are one of the major causes of food-borne illnesses worldwide and pose an important public health problem. On a global scale, Salmonella accounts for approximately three billion human infections each year. The World Health Organization $(\mathrm{WHO})$ has estimated that typhoid fever contributes to 21.7 million illnesses per year (217,000 deaths), with paratyphoid fever accounting for 5.4 million of these cases. ${ }^{(1)}$

Many serotypes of the Salmonella species have been identified. They can be broadly divided into two groups: typhoidal and non-typhoidal. The typhoidal group (e.g. Salmonella enterica serovars Typhi and Paratyphi A and B) causes enteric fever, while the non-typhoidal group (e.g. Salmonella enterica serovar Enteritidis) causes largely uncomplicated enterocolitis.

Although antimicrobial treatments are not indicated for selflimiting uncomplicated Salmonella infections in patients without underlying comorbidities and non-immunocompromised patients, they are necessary for invasive Salmonella infections. ${ }^{(1,2)}$ Without treatment, enteric fever carries a mortality rate of $30 \%$, while appropriate antimicrobial treatment reduces the mortality rate to as low as $0.5 \% .{ }^{(1)}$ At present, due to the widespread emergence of strains resistant to traditional first-line drugs such as ampicillin, chloramphenicol and trimethoprim, current recommendations suggest using fluoroquinolones (i.e. ciprofloxacin) or extendedspectrum cephalosporins (i.e. ceftriaxone) for the treatment of invasive and severe Salmonella infections. ${ }^{(1,2)}$ However, the extensive use of fluoroquinolones has been associated with decreased susceptibility and resistance to this class of drugs. Several clinical trials have demonstrated the superior efficacy of azithromycin compared with fluoroquinolones and extended-spectrum cephalosporins. ${ }^{(3,4)}$ We report the first case of azithromycin-resistant Salmonella isolated in Singapore.

\section{CASE REPORT}

A 65-year-old Chinese man presented to the accident and emergency department with a one-week history of fever, nonproductive cough and exertional dyspnoea with decreased effort tolerance. His past medical history included hypertension, old stroke disease and Parkinson's disease with mild functional limitation. He had no prior travel history in the preceding three months.

The patient was initially treated for bilateral communityacquired pneumonia but subsequently tested positive for human immunodeficiency virus (HIV) infection. His baseline CD4 count was 15 cells $/ \mathrm{mm}^{3}$ and HIV-1 RNA load was 368,501 copies $/ \mathrm{mL}$ $\left(5.57 \log _{10}\right.$ ). Diagnostic bronchoscopy with bronchoalveolar lavage (BAL) was performed and confirmed the diagnosis of Pneumocystis jirovecii pneumonia. He was treated with three weeks of trimethoprim/sulfamethoxazole (TMP/SMX) and started on a highly active antiretroviral therapy regimen consisting of tenofovir, emtricitabine and efavirenz. In view of the patient's significant functional decline, he was transferred to a community hospital for rehabilitation one week later. However, he was transferred back to the acute hospital after two days due to an episode of fever and desaturation, which were attributed to aspiration pneumonia. He was started empirically on piperacillintazobactam. Antibiotic therapy was subsequently escalated to meropenem. Intravenous ganciclovir was started presumptively for the treatment of possible cytomegalovirus (CMV) pneumonitis in view of the positive CMV results for both polymerase chain reaction ( $\mathrm{PCR}$ ) (in the previous $\mathrm{BAL}$ ) and antigenemia tests (CMV cytospin [pp65 antigen] was 1,462 in one million cells). The patient was closely monitored in the high-dependency unit for two days before being transferred to the general ward. No ventilatory support was required.

Since the transfer from the community hospital, the patient had been having loose non-bloody stools with no complaints of abdominal pain, nausea or vomiting. The initial stool culture and Clostridium difficile (CD) toxin test were negative. The patient received meropenem for four days; when his fever resolved and blood cultures yielded no significant bacterial growth, the antibiotic therapy was de-escalated to cefepime. His diarrhoea, however, persisted. Stool cultures repeated four days later grew Salmonella enterica serovar Stanley, which was susceptible to ampicillin, ceftriaxone and carbapenems, with intermediate susceptibility to ciprofloxacin and resistance to TMP/SMX. PCR testing for CD was negative. The stool specimen was also negative for ova, cysts, parasites, cryptosporidium, cyclospora and

${ }^{1}$ Department of Dermatology, ${ }^{2}$ Department of Infectious Diseases, Singapore General Hospital, ${ }^{3}$ Diagnostic Bacteriology, Duke-NUS Graduate Medical School, ${ }^{4}$ Department of Pathology, Singapore General Hospital, Singapore

Correspondence: Dr Phoon Yee Wei, Senior Resident, Department of Dermatology, Singapore General Hospital, Outram Road, Singapore 169608. yeewei1983@hotmail.com 
cystoisospora. To treat the Salmonella enteritis, antibiotic therapy was de-escalated to ceftriaxone, but the patient's diarrhoea persisted despite receiving five days of ceftriaxone. CMV cytospin, repeated after a week of ganciclovir, was 24 in one million cells.

Computed tomography of the abdomen and pelvis showed no significant mural thickening of the colon that was suggestive of colitis. Stool culture, repeated after eight days of antibiotic therapy, again isolated Salmonella Stanley, which showed resistance to ampicillin, TMP/SMX, ciprofloxacin (minimum inhibitory concentration $[\mathrm{MIC}] 2 \mathrm{mcg} / \mathrm{mL}$ ), ceftriaxone (MIC $>256 \mathrm{mcg} / \mathrm{mL}$ ) and azithromycin (MIC $32 \mathrm{mcg} / \mathrm{mL}$ ). The extended-spectrum beta-lactamase (ESBL) confirmatory test was positive, with a CTX-M phenotype. The isolate was susceptible to only carbapenems. Upon retesting, the first Salmonella Stanley isolate was also found to be resistant to azithromycin (MIC $64 \mathrm{mcg} / \mathrm{mL}$ ). Susceptibility testing was performed using the Clinical and Laboratory Standards Institute (CLSI) disk diffusion method. MIC was determined using Etest (bioMérieux, Marcy I'Etoile, France) and also interpreted according to the CLSI document (except for azithromycin, for which there are no CLSI criteria). ${ }^{(5)}$ Repeated CD PCR was negative. Additional blood cultures showed no bacterial growth. The patient was treated with three days of ertapenem, after which his diarrhoea resolved. He was discharged after physiotherapy and caregiver training. The patient was also advised about adherence to antiretroviral therapy.

\section{DISCUSSION}

We report the first clinical case of azithromycin-resistant Salmonella isolated in Singapore. The isolate also showed resistance to ciprofloxacin and ceftriaxone. We postulate that strains of Salmonella with increasing resistance were isolated in subsequent stool cultures either due to infection with a mixed population of Salmonella or a de novo mutation as a result of selective pressure exerted by broad-spectrum antibiotics.

Prior to this case, there have been sporadic reports of strains of Salmonella Paratyphi A with azithromycin resistance in other parts of the world, especially in developing countries such as India and Cambodia, where salmonellosis is endemic. ${ }^{(6-9)}$ Ciprofloxacin has been the first-line antibiotic of choice for the treatment of Salmonella infections since the widespread emergence of multidrug-resistant Salmonella Typhi; in the 1980s, the bacterium was found to be resistant to chloramphenicol, ampicillin and cotrimoxazole. ${ }^{(1,2)}$ However, from the 1990s, there has been a rising incidence of treatment failures, with decreased ciprofloxacin susceptibility (DCS) and fluoroquinolone resistance in Salmonella isolates, possibly due to pervasive and inappropriate antibiotic prescription practices. ${ }^{(1)}$ The mechanism of fluoroquinolone resistance occurs via a mutation in DNA gyrase. Indeed, this is a cause for concern, especially in endemic areas where ciprofloxacin remains the mainstay of treatment. ${ }^{(1)}$ The emergence of DCS and fluoroquinolone resistance has prompted the increased use of third-generation cephalosporins in the treatment of invasive Salmonella infections. Recently, there have been sporadic reports of a high level of resistance to ceftriaxone, as CTX-M-15 ESBLs were found in Salmonella. ${ }^{(10)}$
Azithromycin is an azalide antimicrobial agent that has been demonstrated in clinical trials to be equivalent or superior to chloramphenicol, fluoroquinolones and extended-spectrum cephalosporins for the management of uncomplicated typhoid fever. ${ }^{(3,4)}$ In vitro studies have demonstrated azithromycin's excellent tissue penetration and ability to achieve high intracellular concentrations (> 100 times higher than serum) in macrophages and neutrophils, coupled with a long half-life of $2-3$ days. ${ }^{(11,12)}$ Thus, it has been increasingly favoured for the management of bacillary dysentery and invasive non-typhoidal Salmonella infections. However, the clinical breakpoints for Salmonella and azithromycin have yet to be defined. The European Committee on Antimicrobial Susceptibility Testing states that isolates with an $\mathrm{MIC}<16 \mu \mathrm{g} / \mathrm{mL}$ for azithromycin should be considered as wild-type organisms that are responsive to treatment. ${ }^{(13)}$ In previous studies of typhoidal Salmonella isolates, MICs for azithromycin ranged from $4-64 \mu \mathrm{g} / \mathrm{mL} .{ }^{(14)}$

Macrolide resistance has often been reported due to the expression of macrolide 2'-phosphotransferase encoded by the $m p h(\mathrm{~A})$ gene. ${ }^{(10)}$ It has been hypothesised that Escherichia coli may constitute a major reservoir from which plasmid-mediated transfer of resistance genes occur. ${ }^{(14)}$ In the present case, molecular analysis for $m p h(\mathrm{~A})$ gene in the Salmonella Stanley isolate was negative. Nonetheless, other genes known to be associated with macrolide resistance have yet to be looked into: $\mathrm{erm}$ genes are responsible for methylases causing target site modification; ere(A) and $\operatorname{ere}(\mathrm{B})$ genes encode for esterases for macrolide inactivation; and $m e f(A)$ and $m s(A)$ genes cause acquisition of efflux pumps. ${ }^{(15)}$

Azithromycin is a useful alternative to ciprofloxacin for outpatient treatment of uncomplicated salmonellosis whose strains have demonstrated reduced susceptibility to fluoroquinolones. The isolation of azithromycin-resistant Salmonella in Singapore is a worrying development, especially in the absence of recent travel in the present case, as it suggests a locally acquired isolate. Recurrent salmonellosis is an AIDS-defining illness. The emergence of multidrug-resistant Salmonella is possibly the result of an increased use of antibiotics, especially macrolides, TMP/ SMX and cephalosporins, both for the treatment of common infections and as a prophylaxis for HIV-positive patients with low CD4 counts (i.e. azithromycin for Mycobacterium avium complex and TMP/SMX for Pneumocystis jirovecii prophylaxis).

In India, several isolates of Salmonella displaying a high level of resistance to azithromycin have been identified.(1) Paradoxically, there has been newfound susceptibility to older antibiotics (ampicillin, cotrimoxazole, chloramphenicol), leading to speculation about antibiotic recycling. ${ }^{(1)}$ The trend toward an increasing emergence of extended beta-lactamases and strains resistant to azithromycin will greatly limit therapeutic options, leaving only carbapenems and tigecycline as secondary antimicrobial drugs.

The high prevalence of foodborne salmonellosis worldwide makes it a significant public health problem. Good food hygiene and practices are undoubtedly important in decreasing the rates of Salmonella infections. With the current ubiquitous antibiotic prescription practices, antimicrobial resistance patterns 
will no doubt continue to evolve. At present, azithromycin remains a viable alternative to ciprofloxacin for the treatment of uncomplicated typhoid fever and invasive non-typhoidal salmonellosis in the community, but it must be used with care to prevent rapid development of widespread resistance. Further research is also needed to determine the clinically relevant breakpoints of azithromycin by reviewing the treatment outcome of azithromycin in relation to its MICs.

In conclusion, the emergence of azithromycin-resistant Salmonella is a worrying phenomenon. We advocate testing for azithromycin susceptibility in ciprofloxacin-resistant cases and high-risk groups, such as the immunocompromised (e.g. HIV patients) and travellers from endemic areas. In addition, local susceptibility data should be collected and considered when selecting empirical antibiotics for cases of suspected Salmonella infections.

\section{REFERENCES}

1. Harish BN, Menezes GA. Antimicrobial resistance in typhoidal salmonellae. Indian J Med Microbiol 2011; 29:223-9.

2. Hohmann EL. Nontyphoidal salmonellosis. Clin Infect Dis 2001; 32:263-9.

3. Butler T, Sridhar CB, Daga MK, et al. Treatment of typhoid fever with azithromycin versus chloramphenicol in a randomized multicentre trial in India. J Antimicrob Chemother 1999; 44:243-50.

4. Chinh NT, Parry CM, Ly NT, et al. A randomized controlled comparison of azithromycin and ofloxacin for treatment of multidrug-resistant or nalidixic acid-resistant enteric fever. Antimicrob Agents Chemother 2000; 44:1855-9.

5. Clinical and Laboratory Standards Institute. Performance Standards for Antimicrobial Susceptibility Testing; Twenty-Third Informational Supplement. Wayne: CLSI, 2013.

6. Molloy A, Nair S, Cooke FJ, et al. First report of Salmonella enterica serotype paratyphi A azithromycin resistance leading to treatment failure. J Clin Microbiol 2010; 48:4655-7.

7. Rai S, Jain S, Prasad KN, Ghoshal U, Dhole TN. Rationale of azithromycin prescribing practices for enteric fever in India. Indian J Med Microbiol 2012; 30:30-3.

8. Vlieghe ER, Phe T, De Smet B, et al. Azithromycin and ciprofloxacin resistance in Salmonella bloodstream infections in Cambodian adults. PLoS Negl Trop Dis 2012; 6:e1933.

9. Hassing RJ, Goessens WH, van Pelt W, et al. Salmonella subtypes with increased MICs for azithromycin in travelers returned to The Netherlands. Emerg Infect Dis 2014; 20:705-8.

10. Le Hello S, Harrois D, Bouchrif B, et al. Highly drug-resistant Salmonella enterica serotype Kentucky ST198-X1: a microbiological study. Lancet Infect Dis 2013; 13:672-9.

11. Lalak NJ, Morris DL. Azithromycin clinical pharmacokinetics. Clin Pharmacokinet 1993; 25:370-4

12. Panteix G, Guillaumond B, Harf R, et al. In-vitro concentration of azithromycin in human phagocytic cells. J Antimicrob Chemother 1993; 31 Suppl E:1-4.

13. Leclercq R, Cantón R, Brown DF, et al. EUCAST expert rules in antimicrobial susceptibility testing. Clin Microbiol Infect 2013; 19:141-60.

14. Sjölund-Karlsson M, Joyce K, Blickenstaff K, et al. Antimicrobial susceptibility to azithromycin among Salmonella enterica isolates from the United States. Antimicrob Agents Chemother 2011; 55:3985-9.

15. Phuc Nguyen MC, Woerther PL, Bouvet M, et al. Escherichia coli as reservoir for macrolide resistance genes. Emerg Infect Dis 2009; $15: 1648-50$ 